\title{
ORBIT STABILIZATION IN SPRING-8 STORAGE RING
}

\author{
N. Kumagai, H. Ohkuma, K. Soutome, M. Takao, H. Tanaka, JASRI / SPring-8 \\ 323-3, Mihara, Mikazuki-cho, Sayo-gun, Hyogo, 679-5198 JAPAN
}

\begin{abstract}
Stability of electron orbit is one of the most important properties to achieve brilliant photon beams for third generation synchrotron radiation sources. In the design of a SPring-8 storage ring, many ideas for orbit stabilization were thus considered. Consequently, without any correction, electron orbit stability of about $50 \mu \mathrm{m}$ per day is obtained. Since a main part of the orbit movement is slowly varying components in time, we are developing both a periodic correction of electron beam energy and that of global orbit to realize the orbit stability of a few $\mu \mathrm{m}$ at source points along the ring. Up to now, we have achieved the stability at each insertion device within several microns per day in an amplitude of the main harmonic of the orbit movement and one correction scheme is now routinely used in user operation.
\end{abstract}

\section{INTRODUCTION}

For highly brilliant synchrotron radiation sources, stability of electron orbit is crucial to achieve brilliant photon beams averaged in certain measurement time. Toward the orbit stability of a few $\mu \mathrm{m}$, perturbation sources for the orbit movement were suppressed as much as possible in the storage ring design. Items considered $[1,2]$ are:

- Construction of a machine tunnel on a hard and stable rock bed.

- Adoption of double compartment structure to make the machine tunnel free from the changes of ambient temperature and sunshine.

- Stabilization of room temperature in a machine tunnel within 1 degree centigrade.

- Stabilization of cooling water within \pm 1 degree centigrade.

- Insertion of thermal insulation between a magnet yoke and coils.

- Optimization of cooling water stream-pass to reduce the peak temperature of magnet coils.

- Reduction of heat loss of magnet power cables and of thermal invasion from both the power cables and cooling water pipes to magnets and magnet girders.

- Removal of vibration sources like a chiller from a machine tunnel and suppression of the magnitude of vibrations by using vibration proof foundation, etc.

- Suppression of the propagation of vibrations to magnet and magnet girders.

- Increment on mechanical hardness of magnet girders.

- Reduction of current ripple of magnet power supplies.

To the remaining orbit movement even after the above treatment, a suitable correction of closed orbit distortion (COD) is applied in accordance with a periodicity of the movement. At present, we have introduced one periodic COD correction scheme to suppress slowly varying components of the COD. We are also preparing to

Corresponding Author, Email: tanaka@spring8.or.jp ntroduce periodic adjustment of an RF frequency to reducie the systematic orbit drift by stabilizing energy of the circulating electron beams. Here, we describe the orbit movement in the ring without any correction and then show our periodic correction scheme and obtained results.

\section{ORBIT MOVEMENT IN SPRING-8 STORAGE RING}

\subsection{Slow Orbit Movement}

Figure $1(\mathrm{~A})$ and (B) show respectively the slow changes of the horizontal and vertical orbit at one beam position monitor (BPM) located in the dispersion-free section of \#41 cell. The sampling period of data is 30 seconds. In this example, the maximum horizontal and vertical changes per day are respectively 70 and $50 \mu \mathrm{m}$. As seen in the figure, the changes don't have a clear periodicity of 24 hrs. And also, these are usually not described by a single error source except for a special case in a vertical plane. For several times, we have observed a large change in a vertical plane correlated with hard rainfall. In this case, the vertical orbit distortion is expressed by a single kick in \#8 cell. This shows that the deformation of the ring due to rainfall is localized at \#8 cell, but the mechanism has not been clearly understood yet.

Figure 2 shows a part of the data in Fig.1 (A) by magnifying the data about forty in a time scale. We can see clear periodic movement, of which period is $6 \sim 7 \mathrm{~min}$ and amplitude is about $10 \mu \mathrm{m}$. This is clearly correlated with the temperature change of the cooling water.

Figure 1 (C) shows that the horizontal orbit at one BPM located in the dispersive section of \#22 cell. The maximum change per day is about $50 \mu \mathrm{m}$. This pseudoperiodic change can be clearly explained by earth tide [3]. Variation of the circumference due to earth tide causes the energy change of circulating beams and this systematic error shifts the orbit pseudo-periodically. We therefore expect that this drift can be cured by the proper control of an RF frequency to keep the energy constant.

\subsection{Fast Orbit Movement}

To check the magnitude of fast orbit movement, signal of the BPM located in \#46 cell was measured with the sampling period of $2 \mathrm{msec}$. Since the noise level of this measurement is about $10 \mu \mathrm{m}$, we couldn't see clear signal. From the measurement results we only estimate that the fast orbit movement is the noise level and less. We are now preparing to measure the fast movement more precisely.

\section{PERIODIC COD CORRECTION SCHEME}

\subsection{Correction Scope}

Measured data shows that the magnitude of the slow orbit movement is relatively larger than that of the fast one. We then tried to develop a scheme to correct the slow orbit movement with a period of a minute and longer. 

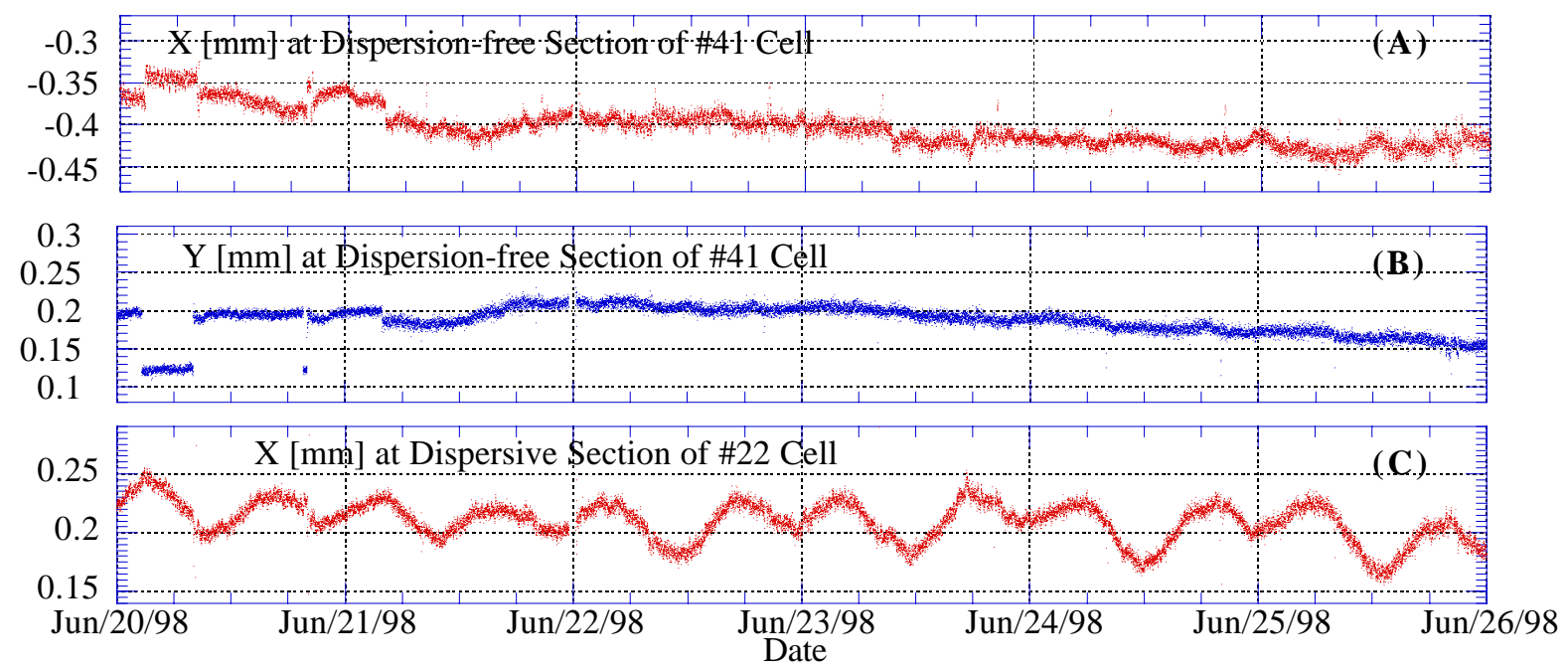

Figure 1: Slow orbit movement for 6 days in the SPring-8 storage ring without any correction. The graphs (A) and (C) show the horizontal orbit movement at one BPM located respectively in dispersion-free and dispersive sections. The (B) shows vertical orbit movement at one BPM located in the dispersion-free section.

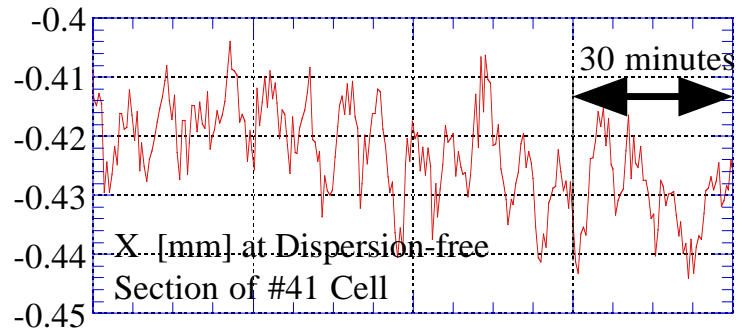

Figure 2: Magnified data of a part of Fig. 1 (A). Two hours data from Jun/25/98 12:00:00 14:00:00 are shown.

At this moment, we correct only harmonic components corresponding to a betatron tune and its satellite harmonics, which are main components of the global movement. Although the accuracy of our BPM system is $4 \sim 5 \mu \mathrm{m}$ in an r.m.s. value [4], the magnitude of random noise is reduced by the square root of the BPM total number through the data integration in harmonic expansion. In the SPring- 8 storage ring, we have 288 BPMs and this noise reduction factor becomes about 17. This means that the BPM random noise is reduced down to $\sim 0.3 \mu \mathrm{m}$ for an estimation of the harmonic components.

\subsection{Synthesis of Correction Target}

Before starting the periodic correction, reference orbit should be set. The orbit is automatically measured with 288 BPMs along the ring and saved in an archive database. Periodically, the correction program subtracts the reference orbit from the latest one and calculates harmonic components of the difference between the two orbits.

For the correction of the global COD, three harmonics corresponding to the tune and tune \pm 1 are used to synthesize target orbit. In harmonic analysis, we use a simple interpolation of the COD data. Although this treatment induces an error of the harmonic expansion especially at high frequencies, this error is corrected by an analytic correction formula at the synthesis of the target orbit.

For the correction of the pseudo-periodic drift due to earth tide, the 0th harmonic component of the horizontal COD is only used.

\subsection{Correction of Global COD}

The period for the correction is a parameter and it is usually set to $1 \mathrm{~min}$. Available steering dipoles in a horizontal and vertical planes are respectively 96 and 281 . All horizontal steering dipoles are in the dispersion-free sections to avoid a cross talk between the corrections of the COD and beam energy. The number of steering dipole used in one correction step is also a parameter. Usually, 24 and 16 are set respectively for the horizontal and vertical corrections.

The best set of steering dipoles are chosen and the strength of each steering dipole is calculated by well known best corrector search algorithm [5]. A coefficient matrix describing the propagation of a steering kick is made of design parameters. Resolution of current setting is $0.15 \mathrm{~mA}$, which is equivalent to $\sim 0.03$ and $\sim 0.015 \mu \mathrm{rad}$ respectively in a horizontal and vertical planes [6].

\subsection{Correction of Pseudo-periodic Orbit Drift due to Earth Tide}

The RF frequency change $\Delta f$ is related to the change of a Oth harmonic component of the horizontal orbit, $a_{0}$ by Eq. 1 obtained by fitting the measured data. The period for the correction is a parameter. In the case where the correction period is $5 \mathrm{~min}$, we have 10 data of the $a_{0}$ every correction period. By using the least square method and Eq. 1, the RF frequency change is estimated to minimize the deviation from the reference. Resolution of frequency setting is $0.1 \mathrm{~Hz}$.

$$
a_{0}[\mathrm{~mm}]=-1.56 \times 10^{-3} \cdot \Delta f[\mathrm{~Hz}]
$$

\section{CORRECTION PEFORMANCE ACHIEVED}

Figure 3 (A) and (B) show the amplitude changes of tune- 
harmonics (51th for Hori. and 16th for Vert.) of the COD respectively without and with the correction of the global orbit distortion. The correction period was set to $1 \mathrm{~min}$. 24 and 16 steering dipoles were used respectively for the horizontal and vertical corrections. The global COD correction controls amplitudes of the horizontal and vertical tune-harmonics, i.e., correction variables within several and a few $\mathrm{mm}$, respectively. Since the tuneharmonic is a main term of the global orbit distortion in each plane, we predict that the global distortion is controlled at each insertion device within the same order as shown in Fig. 3 (B).

Figure 4 (A) and (B) show respectively the slow changes of the horizontal and vertical orbit at one BPM of \#41 cell with the global COD correction. These data represents the local orbit movement under the correction is on. Thickness of each line in the figure is determined by the BPM reproducibility and the slow undulation seems to show the real orbit movement. The stability of about $10 \mu \mathrm{m}$ per day was obtained by this correction, including the local orbit movement. Spurious horizontal and vertical dispersions here are respectively about 2 and $0.1 \mathrm{~cm}$. Even in a horizontal plane, the deviations due to earth tide is only a few $\mu \mathrm{m}$.

Figure 5 shows a result of the correction test for the pseudo-periodic orbit drift by periodically adjusting the RF frequency. The correction period was set to $5 \mathrm{~min}$. The stability of several $\mu \mathrm{m}$ per $5 \mathrm{hrs}$ was obtained for the 0th component of the horizontal orbit.

\section{REFERENCES}

[1] Conceptual Design Report, SPring-8 Project Part 1 Facility Design 1990 [Draft], JAERI-RIKEN SPring-8 Project Team (1991).

[2] N. Kumagai, SPring-8 Information Vol.3 No. 5 (1998) 1 (in Japanese).

[3] S. Date and N. Kumagai, Nucl. Instr. and Meth. A421 (1999) 417.

[4] S. Sasaki, private communication.

[5] B. Autin and Y. Marti, CERN ISR-MA/73-17 (1973).

[6] K. Kumagai, private communication.

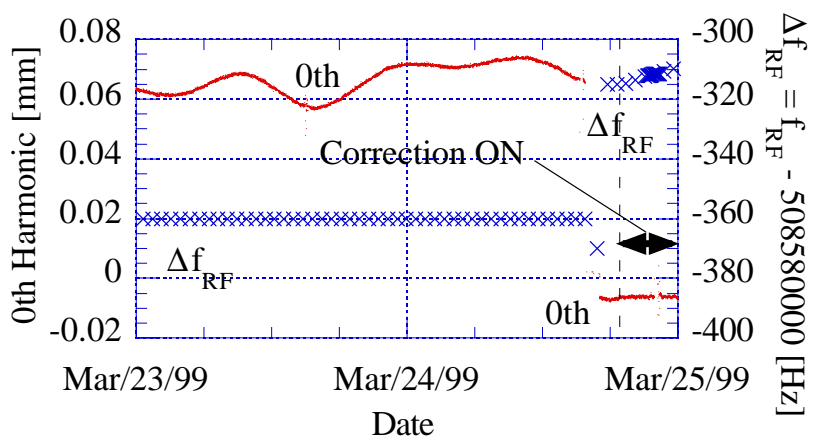

Figure 5: Correction of the 0th harmonic of horizontal COD by adjusting the RF frequency.

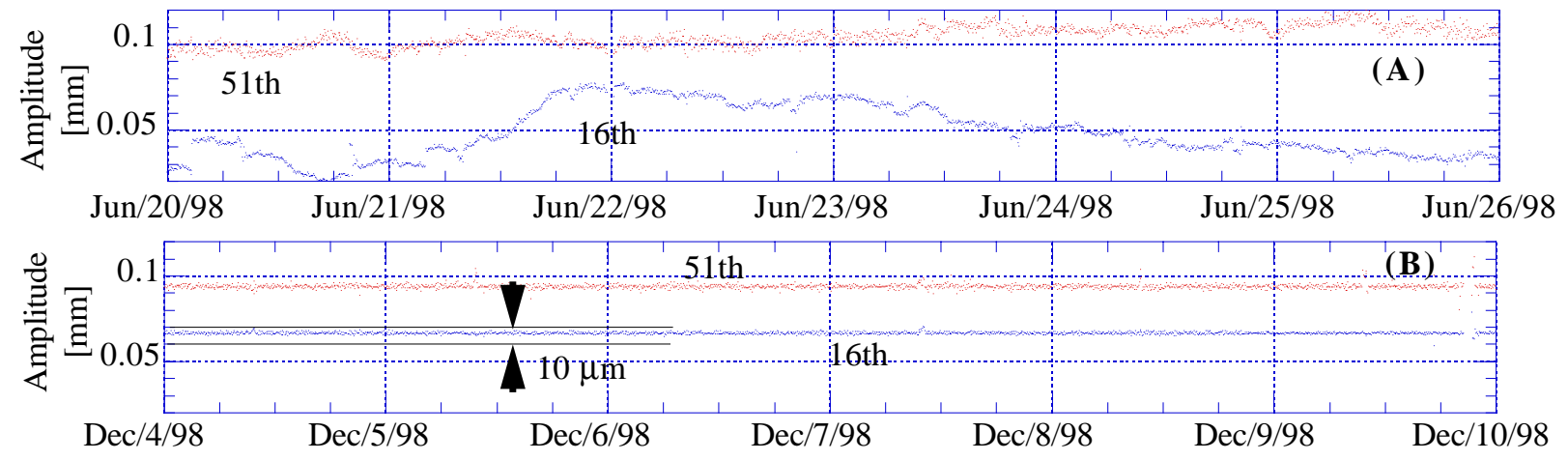

Figure 3: Slow amplitude changes of tune-harmonics (51th for Hori. and 16th for Vert.) of the COD for 6 days. The upper (A) and lower (B) graphs show respectively the changes without and with the periodic correction of the global orbit distortion.

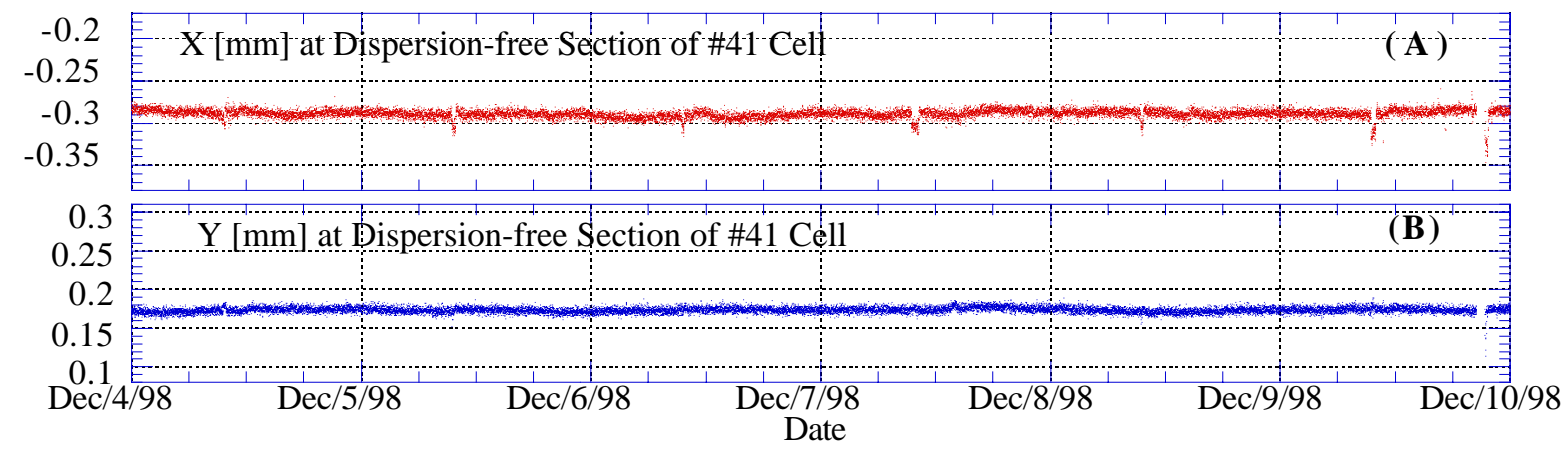

Figure 4: Slow orbit movement for 6 days with the periodic correction of the global orbit distortion. The correction period is 1 minute. The upper (A) and lower (B) graphs show respectively the horizontal and vertical orbit changes at one BPM located in the dispersion-free section of \#41 cell. 